\title{
Energy-efficiency in Decentralized Wireless Networks: A Game-theoretic Approach inspired by Evolutionary Biology
}

\author{
Andrej Gajduk *, Zoran Utkovski,*, Lasko Basnarkov ${ }^{*, \dagger}$ and Ljupco Kocarev ${ }^{*, \dagger, \ddagger}$ \\ * Laboratory for Complex Systems and Networks, \\ Macedonian Academy of Sciences and Arts, Republic of Macedonia \\ \#Faculty of Computer Science, University Goce Delcev, Stip, Republic of Macedonia \\ ${ }^{\dagger}$ Faculty of Computer Science and Engineering, University SS. Cyril and Methodius Skopje, \\ Republic of Macedonia \\ ${ }^{\ddagger}$ BioCircuits Institute, University of California San Diego, USA
}

\begin{abstract}
Energy efficiency is gaining importance in wireless communication networks which have nodes with limited energy supply and signal processing capabilities. We present a numerical study of cooperative communication scenarios based on simple local rules. This is in contrast to most of the approaches in the literature which enforce cooperation by using complex algorithms and require strategic complexity of the network nodes. The approach is motivated by recent results in evolutionary biology which suggest that, if certain mechanism is at work, cooperation can be favored by natural selection, $i$. e. even selfish actions of the individual nodes can lead to emergence of cooperative behavior in the network. The results of the simulations in the context of wireless communication networks verify these observations and indicate that uncomplicated local rules, followed by simple fitness evaluation, can generate network behavior which yields global energy efficiency.
\end{abstract}

\section{INTRODUCTION}

One of the major aspects of future wireless networks is their energy efficiency, which lies at the focus of this work. The provisioning of energy efficient protocols and communication schemes is one of the main challenges in the design of present and future communication networks. The concept of energy efficiency is particularly relevant in emerging heterogeneous networks which, besides the "classical" communication nodes, include various other devices with low-power capabilities, such as sensors and other nodes producing machine-type traffic.

Wireless communication systems have two fundamental properties. The first one is that they receive power decays according to a power law function of the distance between the users, which puts stress on the power consumption. The second important property is the broadcast nature of the wireless communication, which leads to interference between the users. With the increase in the number of subscribers and growth in data traffic in wireless networks, these two features gain in importance and have a strong adverse effect on the network performance in terms of throughput and energy consumption.

The study of the fundamental limits of wireless networks suggests that cooperation among the units could potentially overcome these effects. In this context, techniques such as cooperative diversity [1], [2] and interference alignment [3] have been proposed.

As cooperation is considered to be beneficial in general, most of the present approaches which deal with the aspects of cooperative communications, assume that the network nodes act in a pre-defined way, i. e. their behavior is determined by (usually) centralized network rules. In such systems it is often assumed that the cooperation between the network nodes is beneficial "by default" and no freedom is left to the individual nodes to decide about their involvement in the cooperative act. Besides the networks with central infrastructure, this is also the case in decentralized networks such as ad-hoc networks. One general observation is that the proper functioning of these networks is generally maintained either by enforcing cooperation, or by keeping track of the cooperative behavior which demands intensive computation [4]-[8]. Since cooperation is associated with a cost (usually energy) and requires certain signal processing capabilities (computational complexity), this approach may lead to a "cooperation burden" which can be unreasonably high for some network nodes.

The above described networks, as well as all other present communication networks, can be seen as systems which are mostly "engineered", meaning that they reflect a number of accepted principles of good design. The parts in these systems have known functions and designers attempt to maintain separation of concerns. On the other hand, there are other networks such as biological, social and economic, which evolve over time as a result of the interactions between the system entities and the environment. A formidable remark is that, compared to present communication systems, information storage and processing in living organisms is more efficient by many orders of magnitude, with respect to both information density and energy consumption. Low energy consumption paired with simplicity, efficiency and adaptability seems to be an important objective for information exchange in living organisms.

Motivated by these observations, we will present a gametheoretic approach to the energy efficiency in decentralized wireless networks, which is motivated by the insights obtained 
from evolutionary biology. In particular, we will concentrate on the concept of cooperation and its emergence in communication networks. The model studied in this work considers forwarding the packets of the senders towards the receivers by entities that are cooperators. The choice between cooperative or defective behavior that nodes make in this game theoretic approach is based solely on calculation of their energy consumption. This assumption greatly reduces the computation complexity, as compared with the studies found in the literature [6]-[9]. The simulations show that the population of cooperators persists although the nodes' decisions are selfish, which results in decreased average energy consumption both for the whole population and for the individual nodes.

The remaining of the paper is organized as follows. In Section II the relation between energy efficiency and cooperation is given. Next, in Section III the model studied in this work is described. The experiments and the results obtained are explained in Section IV. Section V concludes this paper.

\section{ENERGY EFFICIENCY AND COOPERATION}

\section{A. Wireless communication networks}

The primary focus of this paper is on the communication in wireless networks. However, the key ideas could be applicable to other communication networks, as well as networks in general, including social and economic networks.

The performance analysis of wireless networks is often based on simplifying assumptions. As a general rule, the cost of establishing cooperation in wireless networks is not properly taken into account when deriving the performance limits of different cooperative schemes [10]. For example, in some scenarios the benefits of cooperation might be overshadowed by the cost of establishing cooperation in the first place. Also, very often a central infrastructure/control is assumed, which is not always the case. In addition, as cooperation comes at a cost for the network users, in a network which lacks centralized control, at certain time instants for some users it might be beneficial to defect, instead of cooperate.

Cooperation in decentralized networks is usually established by complex algorithms [11], [12], which usually promote/enforce cooperation based on reputation tables about the users' behavior. In contrast to the present approaches which rely on complex algorithms in order to enforce cooperation, we are interested in cooperation which emerges as a result of the system evolution. This approach is inspired by recent results in evolutionary biology which suggest that cooperation can emerge and persist in evolving systems, i. e. that cooperation can also be favored by natural selection, if certain mechanism is at work [13], [14].

While we will build on the legacies of communication protocols for establishing cooperation in decentralized networks, our approach differs in one important aspect. Namely, we will not assume cooperation to be beneficial "by default", but we will rather adopt a game-theoretic approach where the network nodes decide whether to cooperate or not based only on their individual fitness, where the fitness is a quantity related to the energy consumption of the individual nodes. We will propose and evaluate different strategies of the individual nodes in terms of the average energy consumption. The focus will be on simple, decentralized strategies which do not require strategic complexity of the involved nodes. This is in the spirit of evolving systems (such as biological) where the cooperative behavior is based on simple rules and its emergence can be understood by relatively simple mechanisms. As we will see, some interesting insights will appear as consequence of the analysis performed in this paper. The most important one is probably the conclusion that, under certain circumstances, there are simple strategies which do not enforce cooperation, but it emerges as a result of the network evolution. These results also serve as an indicator that uncomplicated local, evolutionary-like rules, followed by simple fitness evaluation, can generate network behavior which yields global energy efficiency.

\section{B. Cooperation in biological systems}

Recent results in biology [13]-[16] show that cooperation has played a fundamental role in many of the major transitions in biological evolution and is essential to the functioning of a large number of biological systems. Observations show that cooperative interactions are required for many levels of biological organization ranging from single cells to groups of animals. Human society, as well, is based to a large extent on mechanisms that promote cooperation. In the following paragraphs we will shortly address the concept of cooperation in biology and revisit the candidate mechanisms which explain the emergence and stability of cooperation.

1) Emergence of cooperation in biological systems: It is well known that in unstructured populations, natural selection favors defectors over cooperators. There is much current interest, however, in studying evolutionary games in structured populations and on graphs [13]. In [13] the authors describe a simple rule that is a good approximation for different graphs, including cycles, spatial lattices, random regular graphs, random graphs and scale-free networks. The conclusion is that natural selection favors cooperation, if the benefit of the altruistic act, $b$, divided by the cost, $c$, exceeds the average number of neighbors, $k, b / c>k$. The intuition behind is that in this case cooperation can evolve as a consequence of "social viscosity" even in the absence of reputation effects or strategic complexity.

2) Mechanisms behind the emergence of cooperation: Candidate mechanisms in biology which are able to explain the emergence and stability of cooperation are kin selection, direct reciprocity, indirect reciprocity, network reciprocity, and group selection [14].

Among the candidate mechanisms which promote cooperation based on natural selection, we identify network reciprocity as the most relevant for wireless communication networks. Network reciprocity is a mechanism that aims to explain why cooperation persists in populations where some individuals interact more often [14]. The approach of capturing this effect is evolutionary graph theory, which allows the study of how spatial structure affects evolutionary dynamics. According 
to this model, the individuals of a population occupy the vertices of a graph, where the edges determine who interacts with whom. Additionally, the users are assumed to be plain cooperators and defectors without any strategic complexity. In this setting, the experiments show that cooperators can prevail by forming network clusters, where they help each other.

\section{ENERGY-EFFICIENT DECENTRALIZED WIRELESS NETWORKS}

Many of the most fundamental instances of cooperation in biological systems involve simple entities which lack strategic complexity. This prevents them to adopt strategies that take into account the history of their interactions with other entities. Yet, remarkably, cooperation is present in theses systems, as supported by evidence [17]-[19].

In the context of decentralized wireless networks, we will be interested in design rules which are simple enough to be implemented by communication nodes with limited processing capabilities, yet powerful enough to promote cooperation and to yield global energy efficiency. This is in contrast to most of the present approaches which rely on complex algorithms and reputation tables in order to enforce cooperation in the network [4], [11], [12]. Our objective is to promote cooperation by relying on simple strategies, i.e. by imposing a limited set of rules which mimic the principles of evolution, and let the systems evolve in time.

The question we ask is the following: Can cooperation arise in communication networks by evolution? If yes, which mechanism should be at work? It seems that network reciprocity is a promising candidate for promotion of cooperation in communication networks. Indeed, when wireless networks are described as graphs, an analogy can be drawn with populations which are not well mixed. The reason for this is that, given a power constraint, one user can interact only with the nodes which are in the range of its transmission, forming a cluster of potential cooperators.

In order to investigate the effects of the application of this kind of mechanism to wireless communication networks, we define a relatively simple network model which, however, captures both the essence of wireless communication networks and the graph models used in evolutionary game theory. We simulate the emergence of cooperative behavior in a communication network in order to explore whether rules such as natural selection can favor cooperation in these networks.

\section{A. Network model}

We model the network as a graph where the users represent the nodes and the edges are related to interactions between them. The objective of each network node is to be power efficient, i.e to minimize the amount of power it spends for packet transmission. As in game theory, we assume two types of nodes, cooperators and defectors. Additionally, we make the following assumptions. First, we assume that the power decays as a power law function of the distance to the transmitter (source). Hence, if the transmit power is $P_{T}$, the power received at distance $d$ from the transmitter is

$$
P_{R}=\frac{P_{T}}{K d^{\alpha}},
$$

where $\alpha$ depends on the propagation characteristics of the area (urban, suburban, rural, etc.) and $K$ is an arbitrary constant. Typically, $\alpha$ takes values in the range $2 \leq \alpha \leq 4$. Due to the power law decay, the presence of cooperators might be beneficial with regard to the energy efficiency. For simplicity we assume either one-hop retransmission (in the presence of a cooperator), or direct communication (in the case when there are no cooperators willing to retransmit the packet).

A time division multiple access (TDMA) approach is used where the nodes take turns in transmitting their packets (no frequency reuse). We divide the time scale in time slots of equal duration and assume that one transmitter/receiver pair is activated at random in each time slot. This multiple access scheme is known to be optimal, at least in first approximation, from a minimum energy per bit perspective. Although this assumption simplifies the network analysis, it may be regarded as restrictive, as it does not include interference/collisions. Nevertheless, we expect that this simple scenario will be able to capture the essence of the cooperative behaviour of the users and that, from the perspective of the investigated phenomenon (emergence of cooperation), the simulation results will be a reasonable indicator of the network behaviour in the more general case. A precise study of the phenomenon in the scenario which embraces simultaneous transmission is a topic of current work. Under these assumptions, we will be essentially interested in the total power consumed by the network over time.

Let us say that during one time slot user A needs to transmit to user B and that the power it uses for direct transmission is $P_{D}$. As a result of the propagation effects, the received power at user B is $P_{R}=P_{D} /\left(K d_{A B}^{\alpha}\right)$. We define the signalto-noise ratio at the receiver as $S N R=P_{R} / \sigma^{2}$, where $\sigma^{2}$ is the noise variance. We say that the transmission is successful if the signal-to-noise ratio at the receiver exceeds a certain threshold, $S N R \geq S N R_{0}=P_{R_{0}} / \sigma^{2}$, which is required for reliable reception. In other words, in order to have a successful transmission, the node A should transmit with power $P_{D} \geq K d_{A B}^{\alpha} P_{R_{0}}$. In the first instant, for simplicity, we assume perfect power adaptation (which should be justified in general) and assume that the node A adjusts the transmit power to the distance $d_{A B}$, such that it meets the receive SNR requirement exactly. This is, of course, a simplification, since for this adaptation to work, A should know the network topology (the distance to $\mathrm{B}, d_{A B}$ ) or, at least, to have a feedback from $B$ about the receive SNR such that it can adjust the transmit power $P_{D}$.

We say that a node $C$ is in range of $\mathrm{A}$, or connected to $\mathrm{A}$, if it can "hear" A's transmission to B. Under the assumed power adaptation, a node $\mathrm{C}$ is in the range of $\mathrm{A}$ if $d_{A C} \leq d_{A B}$. On the other hand, the retransmission only makes sense if $d_{C B} \leq d_{A B}$. Hence, potential cooperators will be located in 
the area described by

$$
\begin{aligned}
& d_{A C}<d_{A B} \\
& d_{C B}<d_{A B} .
\end{aligned}
$$

A node $\mathrm{C}$ which fulfills (2) is called intermediate node. The equality in (2) has been left out in order to avoid defining $\mathrm{B}$ as an intermediate node. In the time period when node $\mathrm{A}$ transmits data to node $\mathrm{B}$, there may be several intermediate nodes. In Fig. 1 the intermediate nodes are located in the area enclosed with a dashed line. In order to decrease the transmission cost for user $\mathrm{A}$, we further reduce the region where the possible cooperators are found by introducing a parameter $\nu \in(0,1)$. With this convention, instead of engaging all cooperators from the area defined with (2) in the retransmissions, we account only for those in the area enclosed by full line. This area includes the nodes at reduced distance $\nu \cdot d_{A B}$ from the transmitter.

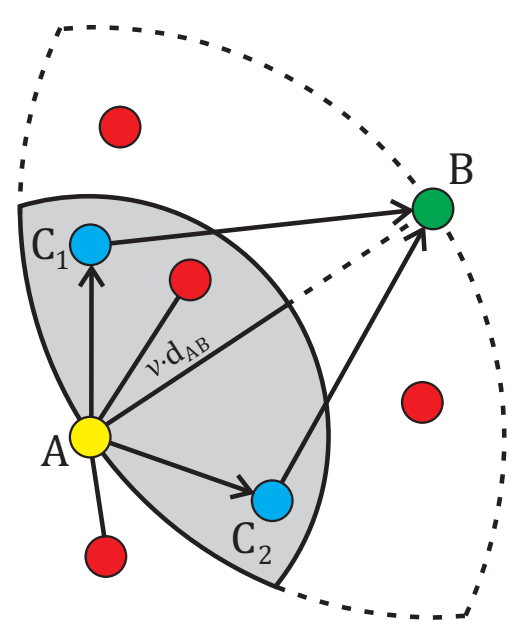

Fig. 1. Typical communication scenario: yellow circle - transmitter ; green circle - receiver; blue circles - cooperators; red circles - nodes that do not participate in the communication; dashed line - the area where intermediate nodes are located; full line - the area where the cooperators for the transmission/receiver pair $\mathrm{A} / \mathrm{B}$ are located;

The relations between the nodes are represented as edges in the graph. We note that this means that each transmission from A to B is associated with one directed network graph. Since in real networks there are simultaneous transmissions between different pairs of users, at each time slot the networks is actually described by a set of different directed graphs, rather then a single graph. Depending on the users activity, the set of graphs also changes over time.

Now, if an intermediate node $\mathrm{C}$ decides to help $\mathrm{A}$ in the transmission, i.e. to cooperate, it will retransmit the signal received from $\mathrm{A}$ to $\mathrm{B}$. The benefit that node $\mathrm{A}$ obtains from the cooperative act of $\mathrm{C}$ is that it can decrease the transmit power to a value lower then the power required for direct transmission, $P_{I} \leq P_{D}$, where the subscript $I$ stands for indirect transmission (transmission when cooperators are involved). In this context, we can define the benefit of the cooperative act as $b=P_{D}-P_{I}$.
In general for a given transmitter/receiver pair there can be multiple cooperators. In this case they can either share the cost for cooperation or let one cooperator pay the overall cost. For simplicity, we propose that only the closest cooperator to B retransmits the signal. Different approaches to cost sharing will be left for future study. Without loss of generality, let $k$ be the index for which $d_{C_{k} B}$ is minimal. As elaborated before, we take that the power received at $\mathrm{B}$ should be exactly the minimal one required for successful transmission, $P_{R_{0}}$. In this case the cost that the cooperator $k$ (the closest one) pays is

$$
P_{C_{k}}=P_{D}\left(C_{k}, B\right)
$$

while the other potential cooperators pay no cost.

Let us now change the perspective and look at the network at time slot $n$ from the viewpoint of a single node (user), node $\mathrm{C}$ for example. For the node $\mathrm{C}$ we distinguish between incoming and outgoing edges as depicted in Fig. 2. The outgoing edges are associated with the nodes which are in the range of $\mathrm{C}$, when $\mathrm{C}$ transmits its own packets. We take that the number of incoming edges to node $C$ is $L$, out of which $J \leq L$ are active (associated with ongoing transmissions). According to this model, the total power that $\mathrm{C}$ spends for cooperation, $P_{C}$, is a function of $J, P_{C}=P_{C}(J)$. On the other hand, the power that $\mathrm{C}$ spends for its own transmission is either $P_{I}$ or $P_{D}$.

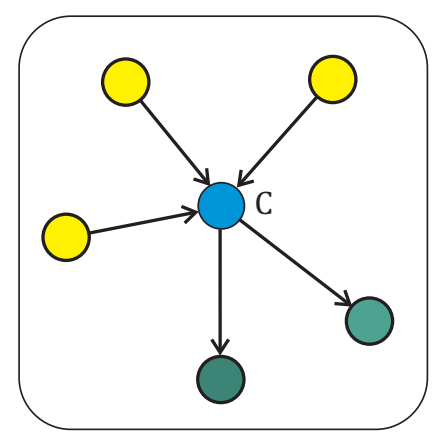

Fig. 2. Incoming and outgoing edges for node C.

If the node $\mathrm{C}$ is in the range of a transmitting node, let us say A for example, then there is an incoming edge to $\mathrm{C}$, outgoing from $\mathrm{A}$. We note that by adapting this model, we allow that at one time slot the node $\mathrm{C}$ can be in the range of several other nodes and also help several of them in the transmission. In reality this can be done by performing a kind of multiplexing at the nodes, for example by using spread-spectrum sequences to distinguish between the different users.

Following the analogy with biology, we will define fitness of the individual nodes. Intuitively, the fitness has to be related with the energy consumption of the individual nodes. Ideally, the appropriate fitness function has to be simple enough to be evaluated locally (possibly without requiring complex processing and memory), but also rich enough to capture the essence of wireless transmission and network dynamics. In addition, we recall that the strategies of the individual nodes (in game-theoretic sense) are adopted with respect to the fitness function. In this sense, the choice of the 
fitness function is related to the game-theoretic analysis of the different strategies.

We will define two discrete time scales, according to which the fitness will be evaluated. The fitness function is evaluated at the end of a block of duration $T$ slots. The network performance is observed over $N$ such blocks (iterations).

Since we have two time scales, we introduce two indices, $t$ and $n$, where $t \in\{0,1, \ldots, T\}$ indicates the time slot and $n \in\{0, \ldots, N\}$ indicates the iteration. The fitness is then a function of $n$ and $t, F=F(n, t)$. We note that, in order to be consistent with the definition of $t$, we denote the initial iteration (of duration $T$ ) as the 0-th iteration. Additionally, we denote the initial fitness as $F(0,0)=F_{0}$.

Now, let us define

$$
\Delta f(n, t)=F(n, t)-F(n, t-1), t=1, \ldots, T,
$$

which measures the difference in the fitness evaluated at two consecutive time instants $t-1$ and $t$, of the $n$-th iteration. In our model, $\Delta f(n, t)$ for the network node $\mathrm{C}$ is defined as

$$
\Delta f(n, t)=-\alpha(1-\beta)\left[P_{D}-P_{I}\right]-\gamma \delta P_{C}(J)
$$

where $\alpha, \beta, \gamma, \delta \in\{0,1\}$ are parameters which indicate packet transmission and the presence of cooperators and defectors. In particular, $\alpha=1$ when $\mathrm{C}$ has a packet to transmit; $\beta=1$ when $\mathrm{C}$ has at least one cooperator as a neighbor; $\gamma=1$ when $\mathrm{C}$ is connected to at least one active node at that time instant; and $\delta=1$ corresponds to $\mathrm{C}$ being a cooperator (otherwise the parameter values are zeros). We note that the above parameters are also functions of $n$ and $t$. However, whenever there is no ambiguity, and in order to simplify the notation, we will skip these indices.

Having introduced $\Delta f(n, t)$, we can define the fitness of the node $\mathrm{C}$ in the following way

$$
\begin{aligned}
F(0,0) & =F_{0}, \\
F(n, t) & =F(n, t-1)+\Delta f(n, t), \\
F(n+1,0) & =F(n, T)
\end{aligned}
$$

where $n=0,1, \ldots, N-1$ and $t=0,1, \ldots, T$. In addition, we define the quantity

$$
\Delta F(n)=F(n, T)-F(n-1, T), n=1, \ldots, N
$$

to be the change of fitness between two consecutive iterations, $n-1$ and $n$. As we will see, this quantity will be relevant for the definition of the different strategies.

\section{B. A Game-theoretic Approach}

Game-theoretic approaches to modeling of phenomena assumes the existence of some quantity - utility, or benefit that units in the system try to maximize. In some scenarios the agents may choose to help the others, i.e. to cooperate this is modeled by the cost they pay for the cooperation. Some agents choose their strategy to be selfish, i.e. they defect, and thus avoid any costs. The cost of cooperative act implies that the cooperators will have smaller fitness than the defectors. Thus natural selection of the fittest favors defectors. However, there are observations and theoretic analyzes of cases when cooperation persists - there is at least a fraction of cooperators present in the population.

In this work we study four different strategies of cooperative behavior of nodes in communication networks. According to our approach we assume that all network nodes adopt the same strategy during the simulations. This approach certainly does not cover some more general scenarios, for example one where the individual nodes are able to choose their strategy at random, or according to some rule. Nevertheless, we expect that the results from our analysis will fairly well indicate the general trend and, as such, will be useful in the evaluation of the fundamental limits of the energy efficiency in decentralized networks.

The first strategy addresses the trivial case when there is no cooperation between the nodes, i. e. all nodes are defectors. We denote this strategy by DEF. The second strategy corresponds to the case where all nodes cooperate and will be denoted as COOP. It corresponds to a centralized scenario where the cooperation is in a way enforced in the network. The main effect we expect from cooperation among the nodes is a decrease in the total energy consumption. The simulation results show that when all nodes cooperate the total energy consumption is reduced by as much as $60 \%$ as opposed to the case when all nodes defect.

As already discussed, for us of greater interest are strategies which are appropriate for the decentralized scenario at hand, where the individual nodes decide whether to cooperate or not based on their individual fitness. In the decentralized scenario that we propose, at the end of each iteration the network nodes decide whether to cooperate or defect in the next iteration. As we do not assume nodes with strategic complexity, we will concentrate on simple strategies. According to this approach, at the end of the $n$-th iteration each node makes the decision only based on the change in the fitness $\Delta F(n)=F(n, T)-$ $F(n-1, T)$, as defined in (7).

We will distinguish between two simple and intuitive strategies for the decentralized scenario. According to the first one, if the node observes an increase in the fitness, $\Delta F(n)>0$, it will retain the previous status in the next iteration. If, on the other hand, the node observes a decrease in the fitness $\Delta F(n)<0$, the node will change its behavior, i. e. a cooperator will become a defector and vice versa. We observe that from the perspective of a single node, the game resembles the repeated prisoner's dilemma [15]. In this regard, the above described strategy corresponds to the well known win-stay, lose-shift and is based on the simple idea of retaining the previous status when the node is doing well, but changing it otherwise. In the remaining of the text we refer to this strategy as WSLS.

According to the other strategy for the decentralized scenario, the node will decide to cooperate in the next iteration if it observes an increase in the fitness, $\Delta F(n)>0$. Otherwise, it will defect. According to this strategy, a defector will become cooperator and a cooperator will stay cooperator, if $\Delta F(n)>0$. Otherwise, the node will choose to defect. We 
note that the increase in fitness reflects the average behavior of the adjacent nodes, in the sense that the reason for the fitness increase is the cooperative behavior of some of the adjacent nodes. In the context of the repeated prisoner's dilemma, this strategy resembles the tit-for-tat strategy which is based on the idea of mimicking the other node(s) behavior in the previous turn. This means that the node will become cooperator only if it observes cooperative behavior of other nodes which is reflected in the increase in the fitness. In the remaining of the text, we will refer to this strategy as TFT.

\section{DESCRIPTION OF THE EXPERIMENT}

\section{A. Simulation setup}

Having defined the different strategies, the aim of the simulations will be to evaluate and compare the performance of all strategies in terms of both the energy consumption of the individual nodes and the global energy consumption in the network. As already discussed, although we would like to see the global energy consumption decreased, we would also like to have the energy consumption distributed as uniformly as possible between the different nodes.

Our simulation is set up as follows. We place $M$ wireless nodes at random in a circle of radius $r$, according to a uniform distribution. Since we are interested only in the relative performance of the different strategies and not in the absolute value of the consumed energy, we normalize the circle radius to a single distance unit, $r=1$. The nodes send their messages during time slots of fixed duration (same for all nodes), where in each time slot exactly one transmitter/receiver pair is activated at random. We group the time slots in blocks of length $T$, where we denote the block of $T$ slots as one iteration. The network behavior is observed over $N$ iterations.

We consider two simulation scenarios. In the first scenario we assume that either all nodes are defectors (DEF strategy), or cooperators (COOP strategy). In the second simulation scenario we start by assuming that in the initial iteration all users are defectors. At the end of the initial iteration we choose one user at random and change its behavior from defector to cooperator. At the end of each next iteration the nodes perform a fitness update and perform a comparison with the fitness at the end of the previous iteration, as given by (7). According to the change of the fitness defined in (7), the users determine their behavior during the next iteration (cooperate or defect) according to the TFT or the WSLS strategy. Once again, we note that in our setup all users follow the same strategy during all iterations.

In both scenarios we are interested in the individual and in the total energy consumption during the $N$ iterations. Since we are interested in the relative performance of the different strategies, for reference we will take the average consumed energy (power) per node in the case when the nodes adopt the DEF strategy. Without loss of generality, we will consider this energy to have value 1 and express the energy consumption of the different strategies with respect to this value.

The simulations were performed for the extreme case of the propagation parameter $\alpha=4$, for which the cooperation is most beneficial in terms of energy consumption. To mimic the simultaneous transmissions present in reality, we have taken a frame length of $T=1000$ consecutive time slots that correspond to 1000 packets. Additionally, the simulations last $N=1000$ iterations. At last, we have considered $N_{t}=1000$ different network topologies obtained by random placement of the nodes in the unit circle with uniform distribution. The large number of repetitions has the effect of smoothing the graphical results. The choice of the numerical values of the simulation parameters are summarized in Table I.

TABLE I

SIMULATION PARAMETERS

\begin{tabular}{l|r||l|r} 
Parameter & Value & Parameter & Value \\
\hline$T$ & 1000 & $r$ & 1 \\
$\alpha$ & 4 & $N$ & 1000 \\
$M$ & 30 & $N_{t}$ & 1000
\end{tabular}

\section{B. Scenario 1: DEF and COOP strategy}

When the users adopt the DEF strategy, a transmitter A communicates with a receiver $\mathrm{B}$ by direct transmission. Hence the cost associated with the transmission is $P_{D}=K d_{A B}^{\alpha} P_{R_{0}}$, where $P_{R_{0}}$ is the minimal receive power necessary for successful transmission, as defined in Section III.

In the case when the users adopt the COOP strategy, for a transmitter/receiver pair $\mathrm{A} / \mathrm{B}$, node $\mathrm{A}$ receives a benefit from the cooperators located in the area defined by (2).

The benefit is reflected in the fact that $\mathrm{A}$ can adopt (reduce) the transmit power to a value $P_{I}=K\left(\nu \cdot d_{A B}\right)^{\alpha} P_{R_{0}}$, where $0<\nu<1$ is a parameter which determines the reduced range of A's transmission. Once fixed, the parameter $\nu$ is kept constant for all nodes and during all transmissions. As we will see, the results from the simulations indicate that the choice of this parameter is particularly important for the energy consumption in the network.

The main effect we expect from cooperation among the nodes is a decrease in the average energy consumption. The results from the simulations show that when all nodes cooperate the average energy consumption is reduced by as much as $60 \%$ as opposed to the case when all nodes defect, as indicated in Table II. Apart from the reduction in average energy consumption, cooperation leads to a more-fair energy consumption among the individual nodes. Indeed, when there is no cooperation the nodes which are located further from the center are at a disadvantage as the average distance to the rest of the nodes is larger compared to the nodes which are located near the center, leading to increased energy consumption for transmission. The introduction of cooperation lessens this imbalance to some extent. An illustration of this effect is shown in Fig. 3. As we can see, the introduction of cooperation balances the amount of energy spent by the individual nodes, and decreases the effect of the network topology on the individual energy consumption. 


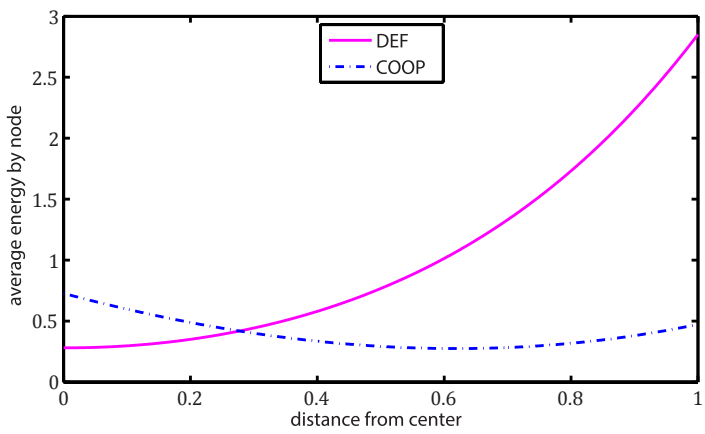

Fig. 3. Average energy consumption for nodes at different distances from the center.

\section{Scenario 2: TFT and WSLS Strategy}

As discussed, in this simulation scenario we start by assuming that in the initial iteration all users are defectors. At the end of the initial iteration we choose one user at random and change its behavior from defector to cooperator.

For one transmitter/receiver pair $\mathrm{A} / \mathrm{B}$, the nodes which fulfill (2) are potential cooperators. If there is a cooperator present, it retransmits the signal to user B. Now, based on the estimation of the receive SNR, the receiver B informs $A$ via feedback (which can also be quantized) that there are cooperators present. With this in mind, the sender A adopts (reduces) the transmit power to a value

$$
P_{I}=K\left(\nu \cdot d_{A B}\right)^{\alpha} P_{R_{0}},
$$

We note that by introducing this heuristics, the sender A does not have to know the identity of the cooperators in each iteration. We recall that after a period of $T$ time slots (one iteration), the nodes calculate their fitness and compare it with the fitness at the end of the previous iteration, as given by (7).

The simulation results indicate that the choice of the parameter $\nu$ is crucial for the energy consumption. We fix this parameter to $\nu=0.39$, a value for which the total energy consumption is approximately minimal, for all strategies, as indicated by the performed simulations.

\section{The effect of cooperation}

Although the simulation results (as expected) show that the COOP strategy yields a minimal total energy consumption among all four strategies, this is not the optimal strategy from the perspective of all of the individual nodes. Indeed, as Fig. 4 indicates, the simulation results show that the adoption of the WSLS strategy yields lower energy consumption for the nodes closer to the center. Further the WSLS strategy yields the most balanced energy consumption as function of the geographical distribution of the nodes. The difference in the balance of energy consumption can be inferred from the standard deviation values given in Table II.

Let us recall that the population starts with only a single cooperator while all other nodes are defectors. The simulations show that with time cooperation spreads through the network. As a single node changes its behavior frequently during a

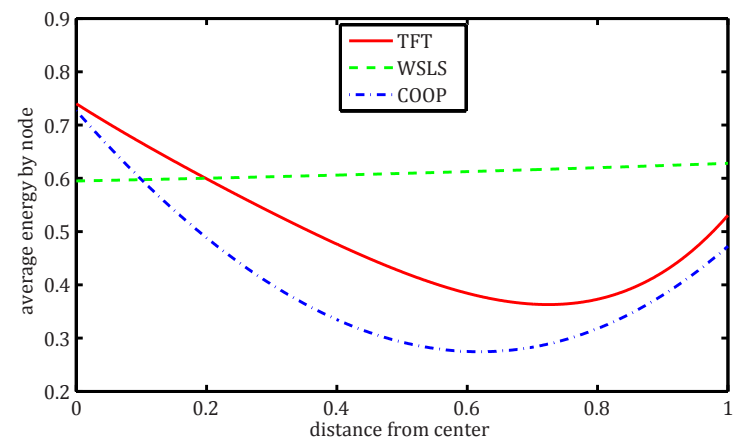

Fig. 4. Average energy consumption for nodes at different distances from the center.

TABLE II

MEAN AND STANDARD DEVIATION OF THE AVERAGE ENERGY CONSUMPTION $E$

\begin{tabular}{l|r|r} 
strategy & $\operatorname{mean}(E)$ & $\operatorname{std}(E)$ \\
\hline DEF & 1.00000 & 0.72150 \\
COOP & 0.39755 & 0.13093 \\
TFT & 0.48858 & 0.11559 \\
WSLS & 0.60966 & 0.01671
\end{tabular}

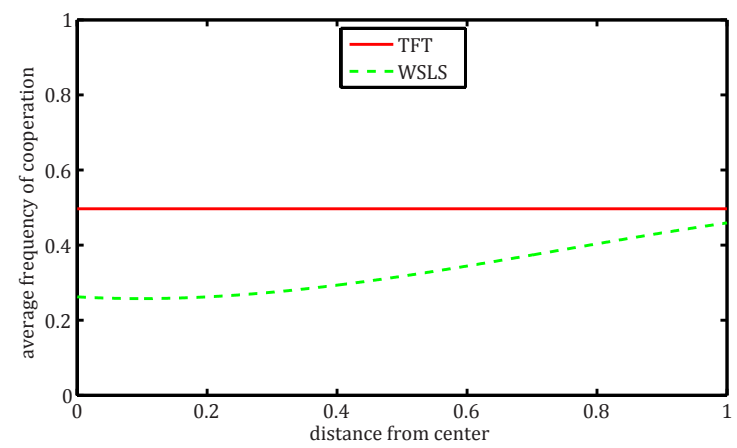

Fig. 5. Average frequency of cooperation for nodes at different distances from the center.

single simulation (depending on the fitness evaluation and on the strategy), we calculate the frequency of cooperation as

$$
f(i)=\frac{\text { number of iterations when node } i \text { is cooperator }}{\text { total number of iterations }}
$$

The results of the simulations show that the frequency of cooperators is fairly constant (around 50\%) for the different placement of the nodes for the TFT strategy, as depicted in Fig. 5. The explanation for this effect is that when the nodes use the TFT strategy, they are more prone to change their behavior from defectors to cooperators and vice versa. We recall that the frequency of cooperators is averaged over 1000 different network topologies, and therefore the constant frequency of cooperators does not come as a surprise. As for the WSLS strategy, it is intuitive to expect that nodes closer to the center will be less incentive to cooperation, compared to those which are further away. 
Fig. 5 can help us understand the balanced energy consumption present in the WSLS scenario. A key observation is that nodes which are positioned closer to the center are cooperators less frequently then others. This reduces their individual energy consumption. Additionally, the absence of cooperators close to the center shifts the cooperation burden to the outer regions. The peripheral nodes thus experience an increase in their energy consumption. Overall, these two effects combined reduce the differences in the individual energy consumption witnessed in TFT and produce the flat curve shown in Fig. 4.

\section{CONCLUSIONS}

We investigated the mechanisms for promotion of cooperation in decentralized wireless networks. The approach was motivated by recent results in evolutionary biology which suggest that cooperation can be favored by natural selection, if certain mechanisms are at work. We modeled the wireless network as a graph, where benefits and costs were associated with the strategy that the network users follow. In gametheoretic spirit, the nodes based their behavior on calculations of their energy spending.

We presented numerical study of cooperative communication scenarios based on simple local rules, which is in contrast to most of the approaches in the literature which enforce cooperation by using complex algorithms and require strategic complexity of the network nodes. We considered four strategies for the users' behavior and observed the energy consumption of the individual nodes and the network in total. In two of the strategies, TFT and WSLS, we considered that the nodes determine their behavior only based on individual fitness. The simulations showed that even selfish decision making of the nodes can lead to emergence of cooperation. These observations served as indicator that uncomplicated local rules, followed by simple fitness evaluation, can generate network behavior which yields global energy efficiency.

We recall that here we adopted the convention that the same strategy was used by all users in all iterations. In a future version of the work, we will consider the case where each of the individual users is allowed to choose its own strategy at every iteration. As discussed, the results from the simulations indicate that, depending on the node distance from the center, distinct nodes could find optimal to follow different strategies. It is expected that this analysis will bring valuable insights in the dependencies between the choice of optimal strategy for the individual users and the network topology.

In addition, it will be interesting to evaluate the network behavior in the case when the nodes have buffers with limited energy capacity, under a particular random arrival process. This is in contrast to the here addressed scenario where we assumed that the nodes have infinite-length buffers. We expect that the adoption of this more realistic assumption will influence both the behavior of the individual nodes and the way energy is consumed in the network. This more general approach also includes the energy harvesting scenario where the nodes harvest energy quants from the environment according to some arrival process.

\section{ACKNOWLEDGMENT}

The authors acknowledge the support of the German Research Foundation (Deutsche Forschungsgemeinschaft - DFG), via the project Li 659/13-1.

\section{REFERENCES}

[1] A. Sendonaris, E. Erkip, and B. Aazhang, "User cooperation diversity, part i: System description," IEEE Transactions on Communications, vol. 51, no. 11, pp. 1927-1938, 2003.

[2] — "User cooperation diversity, part ii: Implementation aspects and performance analysis," IEEE Transactions on Communications, vol. 51, no. 11, pp. 1939-1948, 2003.

[3] V. R. Cadambe and S. A. Jafar, "Interference alignment and degrees of freedom of the $K$-user interference channel," IEEE Transactions on Information Theory, vol. 54, no. 8, pp. 3425-3441, 2008.

[4] S. Zhong, J. Chen, and Y. R. Yang, "Sprite: A simple, cheat-proof, credit-based system for mobile ad-hoc networks," in Twenty-Second Annual Joint Conference of the IEEE Computer and Communications, INFOCOM 2003, vol. 3. IEEE, 2003, pp. 1987-1997.

[5] S. Buchegger, J. Le Boudec et al., "The effect of rumor spreading in reputation systems for mobile ad-hoc networks," in WiOpt'03: Modeling and Optimization in Mobile, Ad Hoc and Wireless Networks, 2003.

[6] Y. Liu and Y. R. Yang, "Reputation propagation and agreement in mobile ad-hoc networks," in Wireless Communications and Networking, WCNC 2003, vol. 3. IEEE, 2003, pp. 1510-1515.

[7] T. Anantvalee and J. Wu, "Reputation-based system for encouraging the cooperation of nodes in mobile ad hoc networks," in IEEE International Conference on Communications, ICC'07. IEEE, 2007, pp. 3383-3388.

[8] J. Mundinger and J.-Y. Le Boudec, "Analysis of a reputation system for mobile ad-hoc networks with liars," Performance Evaluation, vol. 65 no. 3, pp. 212-226, 2008.

[9] S. Buchegger and J.-Y. Le Boudee, "Self-policing mobile ad hoc networks by reputation systems," IEEE Communications Magazine, vol. 43, no. 7, pp. 101-107, 2005.

[10] A. Lozano, R. W. Heath, and J. G. Andrews, "Fundamental limits of cooperation," IEEE transactions on information theory, vol. 59, no. 9, pp. 5213-5226, 2013.

[11] S. Buchegger and J.-Y. Le Boudec, "Performance analysis of the confidant protocol," in Proceedings of the 3rd ACM international symposium on Mobile ad hoc networking \& computing. ACM, 2002, pp. 226-236.

[12] P. Michiardi and R. Molva, "Core: a collaborative reputation mechanism to enforce node cooperation in mobile ad hoc networks," in Advanced Communications and Multimedia Security. Springer, 2002, pp. 107121.

[13] H. Ohtsuki, C. Hauert, E. Lieberman, and M. A. Nowak, "A simple rule for the evolution of cooperation on graphs and social networks," Nature, vol. 441, no. 7092, pp. 502-505, 2006.

[14] M. A. Nowak, "Five rules for the evolution of cooperation," Science, vol. 314, no. 5805, pp. 1560-1563, 2006.

[15] R. Axelrod and W. D. Hamilton, "The evolution of cooperation," Science, vol. 211, no. 4489, pp. 1390-1396, 1981.

[16] J. Cremer, A. Melbinger, and E. Frey, "Growth dynamics and the evolution of cooperation in microbial populations," Scientific reports 2 , Article number 281, no. doi:10.1038/srep00281, 2012.

[17] M. C. Soares, I. M. Côté, S. Cardoso, and R. Bshary, "The cleaning goby mutualism: a system without punishment, partner switching or tactile stimulation," Journal of Zoology, vol. 276, no. 3, pp. 306-312, 2008.

[18] N. J. Mehdiabadi, C. N. Jack, T. T. Farnham, T. G. Platt, S. E. Kalla G. Shaulsky, D. C. Queller, and J. E. Strassmann, "Social evolution: kin preference in a social microbe," Nature, vol. 442, no. 7105, pp. 881-882, 2006.

[19] J. Faaborg, P. Parker, L. DeLay, T. De Vries, J. Bednarz, S. M. Paz, J. Naranjo, and T. Waite, "Confirmation of cooperative polyandry in the galapagos hawk (buteo galapagoensis)," Behavioral Ecology and Sociobiology, vol. 36, no. 2, pp. 83-90, 1995. 